\title{
Frecuencia de portadores nasales de Staphylococcus aureus resistente a meticilina en personal de salud de hospitales de Nicaragua
}

\author{
Mercedes Cáceres ${ }^{1}$
}

Forma de citar

Cáceres M. Frecuencia de portadores nasales de Staphylococcus aureus resistente a meticilina en personal de salud de hospitales de Nicaragua. Rev Panam Salud Publica. 2011;30(6):610-4.

RESUMEN Objetivo. Conocer la frecuencia de portadores nasales de cepas de Staphylococcus aureus resistentes a meticilina (SARM) y el patrón de resistencia antimicrobiana de esas cepas obtenidas de trabajadores de la salud de cuatro hospitales de Nicaragua.

Métodos. Se realizó un estudio descriptivo, transversal, en el período del 1 de junio de 2009 al 30 de septiembre de 2010. Los hisopados nasales de los trabajadores de la salud que aceptaron voluntariamente participar en el estudio fueron cultivados en medio agar base de detección de resistencia a oxacilina (ORSAB). La identificación de los aislados de S. aureus se realizó por métodos cotidianos y la resistencia a meticilina se determinó por la presencia del gen mecA con la técnica de reacción en cadena de polimerasa. El patrón de resistencia antimicrobiana se detectó por difusión en disco. Cada participante firmó un consentimiento informado con anterioridad a la toma de la muestra.

Resultados. Participaron en el estudio 569 trabajadores de la salud, de los cuales 208 eran del hospital de León, 155 de dos hospitales de Chinandega y 206 del de Managua. La frecuencia de portadores nasales de SARM fue de 9,6\% en León, 11,6\% en Chinandega y 6,7\% en Managua. El perfil de resistencia de las cepas SARM fue similar en los cuatro hospitales y todas las cepas fueron sensibles a vancomicina. Del total de cepas SARM aisladas, $15 \%$ fueron multirresistentes. El porcentaje de resistencia a eritromicina fue el más alto, seguido del de clindamicina. Conclusiones. Los resultados del estudio se pueden considerar una advertencia sobre la circulación de cepas SARM entre el personal de salud de los hospitales participantes y aportan información relevante en relación al perfil de resistencia de las cepas SARM.

Palabras clave Farmacorresistencia bacteriana; Staphylococcus aureus; Staphylococcus aureus resistente a meticilina; personal de hospital; Nicaragua.

La resistencia a los antimicrobianos es un problema de salud pública importante, que ha llegado a un punto crítico en muchos hospitales de todo el mundo. Las cepas de Staphylococcus aureus resistente

\footnotetext{
Universidad Nacional Autónoma de NicaraguaLeón, Facultad de Ciencias Médicas, Departamento de Microbiología y Parasitología, León, Nicaragua. La correspondencia debe dirigirse a Mercedes Cáceres. Correo electrónico: merkaceres2001@yahoo.com.mx
}

a meticilina (SARM) son de los agentes patógenos más importantes como causa de infecciones hospitalarias (1). Por otra parte, ese microorganismo es parte de la flora normal de la piel y las mucosas de individuos sanos, aunque también es el agente patógeno causante del aumento creciente de la morbimortalidad por infecciones nosocomiales en todo el mundo. Los portadores nasales de cepas de $S$. aureus tienen un papel significativo en la transmisión del microorganismo. Particularmente en los hospitales, la trasmisión de la bacteria de los pacientes al personal de salud y viceversa es determinante en la génesis de infecciones por cepas de $S$. aureus, ya que la colonización nasal de trabajadores de la salud y pacientes normalmente precede a la infección intrahospitalaria por esta bacteria (2).

La resistencia de las cepas de $S$. aureus a meticilina es primeramente mediada 
por una proteína fijadora de penicilina (PBP) con baja afinidad conocida, como la PBP2a codificada por un gen cromosómico denominado mecA. Se han detectado otros mecanismos de resistencia, como la hiperproducción de betalactamasa, no obstante, en la propagación hospitalaria la presencia de portadores de SARM gen mecA positivo tienen una participación central (3).

El objetivo de este estudio fue conocer la frecuencia de portadores nasales de cepas de $S$. aureus resistente a meticilina y el patrón de resistencia antimicrobiana de las cepas SARM aisladas de trabajadores de la salud de cuatro hospitales nicaragüenses.

\section{MATERIALES Y METODOS}

Se realizó un estudio descriptivo de corte transversal, durante el período del 1 de junio de 2009 al 30 de septiembre de 2010. Las muestras se obtuvieron del personal de salud del hospital Dr. Oscar Danilo Rosales Argüello, de la ciudad de León; los hospitales España y Mauricio Abdalah, de la ciudad de Chinandega, cuyos datos se sumaron y analizaron conjuntamente, y el hospital Dr. Fernando Vélez Paiz, de la ciudad de Managua. Todos son hospitales de segundo nivel que, en 2010, según el Ministerio de Salud de Nicaragua, empleaban 998, 780 y 658 personas en León, Chinandega y Managua, respectivamente. Estos números incluyen personal médico, de enfermería, de laboratorio, de limpieza y administrativo.

La población de estudio estuvo constituida por todos los trabajadores de la salud de los hospitales participantes. La muestra incluyó un total de 560 trabajadores de la salud de esos hospitales constituida por conveniencia y participación voluntaria.

Criterio de inclusión: trabajador de la salud en funciones en uno de los hospitales incluidos en el estudio y que acepta participar voluntariamente mediante la firma de un consentimiento informado.

Criterio de exclusión: trabajador de la salud que no firma el consentimiento informado antes de la toma de la muestra del hisopado nasal o tiene funciones administrativas en el hospital.

\section{Recolección, transporte y procesamiento de las muestras}

Se tomó muestra de las fosas nasales a todos los trabajadores de la salud que cumplieron con los criterios de inclusión. La muestra se tomó de cada una de las fosas nasales utilizando el mismo hisopo de algodón, que fue transportado en medio de transporte Amies agar gel sin carbón (108 - COPAN) al Laboratorio del Departamento de Microbiología de la Facultad de Ciencias Médicas de la Universidad Nacional Autónoma de Nicaragua de León, donde fue inoculado en un medio agar base de detección de resistencia a oxacilina (ORSAB) de Oxoid ${ }^{\circledR} \mathrm{e}$ incubado por 24 a 48 horas a $37^{\circ} \mathrm{C}$.

Todo crecimiento bacteriano en medio ORSAB que mostró color azul fue considerado resistente a meticilina; la identificación de los aislamientos de $S$. aureus se realizó por tinción de Gram, prueba de coagulasa y ADNasa.

La susceptibilidad de los aislados a los antimicrobianos se determinó por el método de difusión en disco de KirbyBauer, según las normas del Instituto de Estándares Clínicos y de Laboratorio (CLSI, por su sigla en inglés) de 2005. Se evaluó la susceptibilidad a clindamicina, eritromicina, ciprofloxacina, trimetoprima/sulfametoxazol, gentamicina $y$ vancomicina (4).

\section{Detección del gen mecA mediante reacción en cadena de polimerasa (PCR)}

Extracción del ADN. Se realizó a partir de un cultivo de 24 horas; en un tubo Eppendorf se suspendieron de 2 a 3 colonias de la bacteria en $120 \mu \mathrm{l}$ de agua destilada estéril. Se sometió a ebullición por 10 minutos y luego se centrifugó por 5 minutos a $8000 \mathrm{rpm}$; se recuperó el sobrenadante en un tubo Eppendorf estéril. Se utilizaron $2 \mu \mathrm{l}$ de sobrenadante como molde ADN para la PCR. Amplificación del ADN: Se tomaron 2 $\mu l$ del sobrenadante de la muestra en el tubo que contenía una mezcla de tapón, magnesio, dNTP, TAQ polimerasa, nucleótido, tapón e iniciador $(23 \mu \mathrm{l})$, a un pH de 8,3. Los iniciadores utilizados para la amplificación de una región altamente conservada del gen mecA fueron: $\operatorname{mec} A$ $1=5^{\prime}$-GCA ATC GCT AAA GAA CTA AG-3' y mecA 2 = 5'-GGG ACC AAC ATA ACC TAA TA-3'. Los iniciadores NUC1 y NUC 2 permitieron obtener 222 y 281 pares de bases o pb, respectivamente. La amplificación se realizó con un termociclador Mastercycler ${ }^{\circledR}$ personal (Eppendorf, Alemania) según el siguiente esquema: desnaturalización a $95{ }^{\circ} \mathrm{C}$ por 7 minutos, seguido de 30 ciclos a $95^{\circ} \mathrm{C}$ por 10 segundos, $58^{\circ} \mathrm{C}$ por 20 segundos y $72{ }^{\circ} \mathrm{C}$ por 2 minutos, y luego una extensión final a $72{ }^{\circ} \mathrm{C}$ por 5 minutos.

Productos. Los productos amplificados fueron analizados por electroforesis mediante geles de agarosa al $2 \%$, teñidos con bromuro de etidio, corridos a 100 $\mathrm{V}$ durante 45 minutos. El tamaño del amplicón se comparó con ADN de 100 pb (marcador molecular). El ADN fue visualizado utilizando un transiluminador ultravioleta.

Para aplicar la técnica y como controles positivos y negativos, se empleó una cepa SaCCUG35601 resistente a oxacilina y portadora del gen mecA y la cepa ATCC 25923, respectivamente (5).

Consideraciones éticas. Se elaboró un formulario para obtener el consentimiento informado. En él se indicaban los objetivos de la investigación, el procedimiento de recolección de muestra (hisopado nasal), y el tratamiento confidencial de la información. Cada participante recibió un sobre cerrado con los resultados de su hisopado en relación con su calidad de portador nasal de cepas de $S$. aureus resistente a meticilina (negativo o positivo según el caso).

El protocolo fue aprobado por el Comité de Bioética para Investigación de la Facultad de Ciencias Médicas, UNAM, León. También se obtuvo permiso para realizar el estudio de los directores de cada uno de los hospitales participantes.

\section{RESULTADOS}

Un total de 569 trabajadores de la salud aceptaron participar voluntariamente en el estudio, provenientes de: el hospital de la ciudad de León, 208; los hospitales de la ciudad de Chinandega, 155, y el hospital de Managua, 206. Entre estos, se identificó un total de 20 portadores nasales de SARM en el hospital de León, 18 en los de Chinandega y 14 en el de Managua. La frecuencia de portadores nasales para cada hospital y la localización según servicio se describe en el cuadro 1, en el que puede observarse que los hospitales con el mayor porcentaje de portadores nasales fueron los de Chinandega. Se destaca el importante número de portadores nasales de SARM en el servicio de cuidados intensivos.

La figura 1 muestra los resultados del análisis molecular (PCR) mediante el cual se identificaron las cepas resisten- 
CUADRO 1. Frecuencia de portadores nasales de aislamientos de Staphylococcus aureus resistentes a meticilina en personal de salud, según servicio y hospital, Nicaragua, 2009-2010

\begin{tabular}{|c|c|c|c|c|c|c|c|c|c|}
\hline \multirow[b]{3}{*}{ Servicio } & \multicolumn{9}{|c|}{ Hospital } \\
\hline & \multicolumn{3}{|c|}{$\begin{array}{l}\text { Dr. Oscar Danilo Rosales, } \\
\text { León }\end{array}$} & \multicolumn{3}{|c|}{$\begin{array}{c}\text { España y Mauricio Abdalah, } \\
\text { Chinandega }\end{array}$} & \multicolumn{3}{|c|}{$\begin{array}{l}\text { Dr. Fernando Vélez Paiz, } \\
\text { Managua }\end{array}$} \\
\hline & No. & Casos & $\%$ & No. & Casos & $\%$ & No. & Casos & $\%$ \\
\hline Medicina interna & 52 & 2 & 15 & 1 & 6,7 & 50 & - & - & \\
\hline Pediatría & 38 & 4 & 10,5 & 20 & 2 & 10,0 & 23 & 3 & 13,0 \\
\hline Cirugía & 29 & 3 & 10,3 & 12 & 1 & 8,3 & 5 & - & - \\
\hline Ginecología y Obstetricia & 27 & 1 & 3,7 & 30 & 2 & 6,7 & 35 & 5 & 14,3 \\
\hline Unidad cuidados intensivos & 17 & 2 & 11,8 & 31 & 7 & 22,6 & 7 & - & - \\
\hline Quirófanos & 20 & 2 & 10,0 & 4 & - & - & 21 & 6 & 28,6 \\
\hline Ortopedia & 13 & 3 & 23,1 & 15 & 2 & 13,3 & 11 & - & - \\
\hline Otros $^{a}$ & 12 & 3 & 25,0 & 28 & 3 & 10,7 & 54 & - & - \\
\hline Total & 20 & 20 & 9,6 & 155 & 18 & 11,6 & 206 & 14 & 6,7 \\
\hline
\end{tabular}

- cantidad cero.

a Personal de limpieza y laboratorio.

FIGURA 1. Aplificación del gen mecA por PCR, aproximadamente de $222 \mathrm{pb}$ y los nuc de 281 pb, Carriles: 1 y 13 marcador molecular de 100 pb, 2 control positivo CCUG35601., 3 control negativo 29213., 4 al 12 y 14 al 24 S. aureus aislados de fosas nasales de trabajadores de la salud, Nicaragua 2009-2010

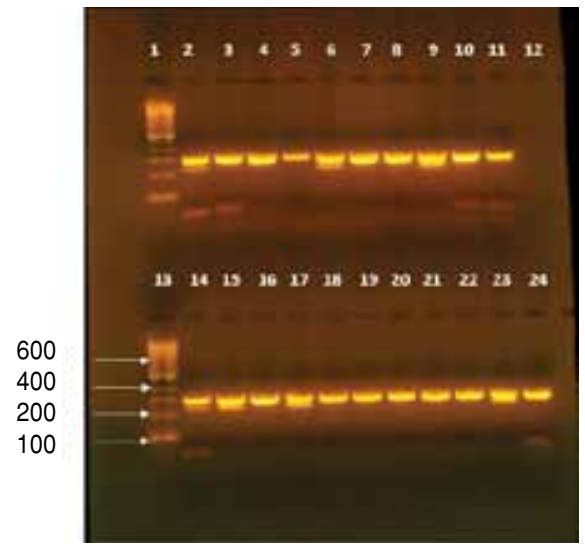

tes a meticilina, que son las portadoras del gen mecA. Se encontró que $15 \%$ de las cepas SARM aisladas de portadores nasales de los hospitales de León y Chinandega eran multirresistentes y que el antimicrobiano de menor utilidad era eritromicina, seguido de clindamicina. No se encontró resistencia a vancomicina. Los porcentajes de resistencia a todos los antimicrobianos analizados se presentan en la figura 2 .

\section{DISCUSIÓN}

Las infecciones causadas por cepas de $S$. aureus resistentes a meticilina constituyen una causa significativa de morbimortalidad en el mundo (6). Por ello,
FIGURA 2. Resistencia antimicrobiana de aislamientos de Staphylococcus aureus resistentes a meticilina obtenidos de portadores nasales en personal de salud, según antibiótico, Nicaragua 2009-2010

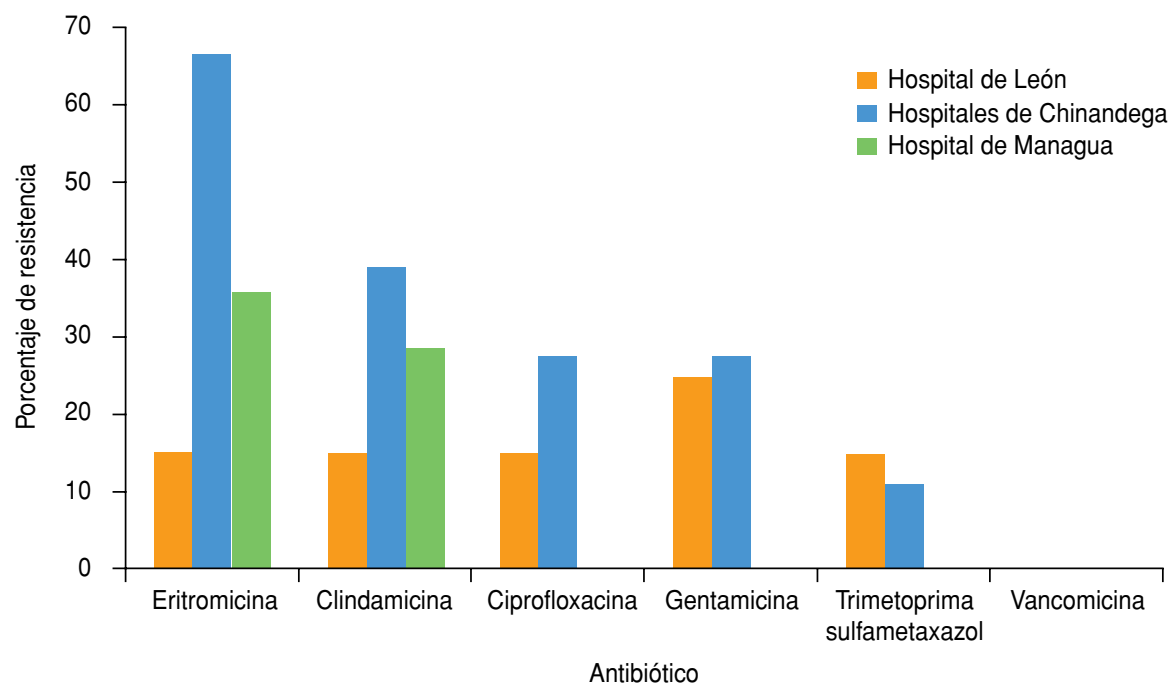

el tratamiento adecuado y oportuno de esas infecciones tiene un impacto importante en los índices de salud. Se ha establecido que un factor de riesgo de estas infecciones es ser portador nasal; a la vez, la portación nasal es el mejor indicador de diseminación del agente, tanto entre los pacientes como entre el personal de salud. La tasa de portación nasal varía de un país a otro, y se ha informado desde $0 \%$ hasta $59 \%$, según un estudio realizado por Albrich y Harbarth, que incluye los resultados de 127 investigaciones referentes a portadores de SARM en trabajadores de la salud (1).

Este es el primer estudio realizado en Nicaragua sobre portadores nasales de SARM entre trabajadores de la salud. Las frecuencias de portadores nasales entre el personal de salud en hospitales de diferentes partes del mundo van desde 4,6 a $5,1 \%$ (1). La frecuencia de portadores nasales de SARM en los hospitales incluidos en este estudio fue de 9,6 en el hospital Dr. Oscar Danilo Rosales de León; 11,6 en los hospitales España y Mauricio Abdalah de Chinandega, y 6,7 en el hospital Vélez Paiz de Managua. Los tres hospitales presentan frecuencias más altas que el promedio informado por Albrich y Harbarth en su análisis de estudios de portadores nasales de SARM (1), pero son similares a las de hospitales de los Estados Unidos de América y la India (7). Cabe destacar que, a pesar de las condiciones higiénicas y sanitarias 
deficientes que presentan los servicios de salud de Nicaragua, la frecuencia de portadores nasales en hospitales nacionales es similar a la de países con un sistema de salud que dispone de los recursos para aplicar medidas de control eficaces.

El aumento de la prevalencia de SARM en todo el mundo, junto con la descripción de cepas con sensibilidad disminuida a los glucopéptidos, que en la práctica se traduce en una pérdida de posibles opciones terapéuticas, hace necesario detectar y controlar la propagación de este tipo de aislamientos. Nicaragua tiene agravantes en relación con el desarrollo de resistencia a los antimicrobianos, ya que es un país donde no se controla la venta de estos fármacos y existe una cultura de automedicación. También existe una práctica de prescripción médica temprana de antibióticos para afecciones que podrían no requerirlos, como son las enfermedades respiratorias y diarreicas, que en su mayoría son de origen viral (8).

El personal de salud de los hospitales que es portador nasal de SARM presenta un riesgo para el propio portador, pero también representa un riesgo de trasmisión de SARM a la comunidad y un aumento potencial de los costos de la atención de los pacientes con infecciones nosocomiales por esta bacteria, especialmente por el aumento de los días de la estancia hospitalaria (9).

Conocer el patrón de resistencia de las cepas SARM encontrado en este estudio es vital para el médico que trata infecciones por estas cepas bacterianas, que son causa importante de infecciones nosocomiales. Es particularmente alto el porcentaje de cepas resistentes a eritromicina, especialmente en los hospitales de Chinandega, aunque dado el número pequeño de cepas, este dato podría no ser significativo. Sí es muy importante considerar el porcentaje de cepas multirresistentes que se encuentran en los tres hospitales estudiados. Este perfil podría explicarse por el hecho de que la resistencia a meticilina se codifica en el gen $m e c A$ y se almacena y transmite en el casete del cromosoma mec (SSC mec). Este vehículo puede llevar también otros genes que confieren resistencia a macrólidos, clindamicina y estreptograminas (3). Además, se sabe que en Nicaragua, al igual que en otros países, la resistencia a los antimicrobianos aumenta día a día. Las cepas de Klebsiella pneumoniae, Escherichia coli productoras de betalactamasa de espectro extendido (BLEE) y $S$. aureus multirresistentes son las causas principales de infecciones hospitalarias. En estudios previos se estableció la importancia de estas bacterias en muestras tanto biológicas como del ambiente en el hospital Dr. Oscar Danilo Rosales Argüello de la ciudad de León, incluido también en el presente estudio (10).

La mayor limitante de este estudio fue la selección de la muestra, que no permite hacer inferencias sobre la población total de trabajadores sanitarios. Otro factor que podría haber afectado los resultados y conclusiones es el no haber incluido el antecedente de uso previo de antibióticos por los participantes.

\section{Conclusiones}

Los hallazgos del presente estudio son una advertencia sobre la circulación de cepas de $S$. aureus portadoras del gen mecA que codifica la resistencia a meticilina entre el personal de salud de los hospitales participantes; asimismo, aporta información relevante en relación al perfil de resistencia a los antimicrobianos de las cepas SARM. Con base en estos resultados del estudio, se recomienda elaborar estrategias que permitan controlar o atenuar la diseminación de estas cepas.

Agradecimiento. A Oscar Arbizú, por su importante colaboración con el estudio en el Hospital Escuela de León y su apoyo con el desarrollo de los análisis moleculares; a Lyrio Calderón y Alina Esquivel por su importante trabajo en el Hospital Dr. Fernando Vélez Paiz de Managua, y a Francil Castillo, Cinnia M. Vilchez y Kathya López por su dedicación con el estudio en los hospitales de Chinandega.

Este estudio fue realizado con fondos de investigación posdoctoral otorgados a la autora por el organismo Sueco, ASDI-SAREC, y la Vice Rectoría de Investigación y Posgrado de la Universidad Nacional Autónoma de NicaraguaLeón.

\section{REFERENCIAS}

1. Albrich W, Harbarth S. Health-care workers: source, vector, or victim of MRSA? Lancet Infect Dis. 2008;8:289-301.

2. Hawkins G. Stewart S, Blatchford O, Reilly J. Should healthcare workers be screened routinely for meticillin-resistant Staphylococcus aureus? A review of the evidence. J Hosp Infect. 2011;77(4):285-9

3. Katayama Y, Ito T, Hiramatsu K. A new class of genetic element, staphylococcus cassette chromosome mec, encodes methicillin resistance in Staphylococcus aureus. Antimicrob Agents Chemother. 2000;44(6):1549-55.

4. Clinical and Laboratory Standard Institute. Approved Standards M2-A8. Performance Standards for Antimicrobial Disk Susceptibility Tests. Approved Standard-8th edition. NCCLS, 2005.

5. Hong F, Goran H. Rapid screening and identification of methicillin-resistant Staphylococcus aureus from clinical samples by selective-broth and real-time PCR Assay. J Clin Microbiol 2003;41(7):2894-9.

6. Durai R, Nq PC, Hoque H. Methicillin-resistant Staphylococcus aureus: an update. AORN J. 2010;91(5):599-606.

7. Vinodhkumaradithyaa A, Uma A, Shirivasan M, Ananthalakshmi I, Nallasivam P, Thirumalaikolundusubramanian P. Nasal carriage of methicillin-resistant Staphylococcus aureus among surgical unit staff. Jpn J Infect Dis. 2009;62(3):228-9.

8. den Engelsen C, van der Werf C, Matute AJ, Delgado E, Schurink CA, Hoepelman AI. Infectious diseases and the use of antibiotics in outpatients at the emergency department of the University Hospital of León, Nicaragua. Int J Infect Dis. 2009;13(3):349-54.

9. Wassenberg MW, de Wit GA, van Hout BA, Bonten MJ. Quantifying cost-effectiveness of controlling nosocomial spread of antibioticresistant bacteria: the case of MRSA. PLoS One. 2010;5(7):e11562.

10. Amaya E, Cáceres M, Hong F, Torres A, Palmgren AC, Nord CE, Weintraub A. Antibiotic resistance patterns in gram-negative and gram-positive bacteria causing septicemia in newborns in León, Nicaragua: correlation with environmental samples. J Chemother. 2010;22(1):25-9.

Manuscrito recibido el 11 de abril de 2011. Aceptado para publicación, tras revisión, el 20 de noviembre de 2011. 
ABSTRACT Objective. To determine the frequency of nasal carriers of strains of methicillinresistant Staphylococcus aureus (MRSA) and the antimicrobial resistance pattern of these strains, obtained from health workers from four hospitals in Nicaragua.

Frequency of nasal carriers of methicillin-resistant Staphylococcus aureus among health workers in Nicaraguan hospitals
Methods. A descriptive cross-sectional study was conducted between 1 June 2009 and 30 September 2010. Nasal swabs were taken from health workers who voluntarily agreed to participate in the study, and were cultured on an oxacillin-resistant screening agar base (ORSAB) medium. The $S$. aureus isolates were identified using ordinary methods, and methicillin resistance was confirmed based on the presence of the mecA gene using the polymerase chain reaction technique. The antimicrobial resistance pattern was detected by the disk diffusion method. Each participant signed an informed consent form before the samples were taken.

Results. A total of 569 health workers participated in the study: 208 from one hospital in León, 155 from two hospitals in Chinandega, and 206 from one hospital in Managua. The frequency of nasal MRSA carriers was $9.6 \%$ in León, $11.6 \%$ in Chinandega, and $6.7 \%$ in Managua. The MRSA resistance profile was similar in the four hospitals, and all the strains were sensitive to vancomycin. Of the total MRSA strains isolated, $15 \%$ were multi-drug resistant. Erythromycin had the highest percentage of resistance, followed by clindamycin.

Conclusions. The results of the study may be regarded as a warning that MRSA strains are circulating among health workers in the participating hospitals. The study also contributes important information regarding the resistance profile of MRSA strains.

Key words Drug resistance, bacterial; Staphylococcus aureus; methicillin-resistant Staphylococcus aureus; personnel, hospital; Nicaragua. 